\title{
Metabolomics comparison of rumen fluid and milk in dairy cattle using proton nuclear magnetic resonance spectroscopy
}

\author{
Jun Sik Eom ${ }^{1, a}$, Eun Tae Kim ${ }^{2, a}$, Hyun Sang Kim ${ }^{1}$, You Young Choi ${ }^{1}$, Shin Ja Lee ${ }^{3}$, \\ Sang Suk Lee ${ }^{4}$, Seon Ho Kim ${ }^{4}$, and Sung Sill Lee ${ }^{1,3, *}$
}

\author{
* Corresponding Author: Sung Sill Lee \\ Tel: +82-55-772-1883, Fax: +82-55-772-1889, \\ E-mail: Iss@gnu.ac.kr \\ ${ }^{1}$ Division of Applied Life Science (BK21Four) \\ Gyeongsang National University, Jinju 52828, \\ Korea \\ 2 National Institute of Animal Science, Rural \\ Development Administration, Cheonan \\ 31000, Korea \\ ${ }^{3}$ Institute of Agriculture and Life Science \\ \& University-Centered Labs, Gyeongsang \\ National University, Jinju 52828, Korea \\ ${ }^{4}$ Ruminant Nutrition and Anaerobe \\ Laboratory, College of Bio-industry Science, \\ Sunchon National University, Suncheon \\ 57922, Korea \\ a These authors contributed equally to the \\ work. \\ ORCID \\ Jun Sik Eom \\ https://orcid.org/0000-0001-5360-0147 \\ Eun Tae Kim \\ https://orcid.org/0000-0001-7486-5638 \\ Hyun Sang Kim \\ https://orcid.org/0000-0002-3422-1990 \\ You Young Choi \\ https://orcid.org/0000-0002-3417-8099 \\ Shin Ja Lee \\ https://orcid.org/0000-0002-4224-1211 \\ Sang Suk Lee \\ https://orcid.org/0000-0003-1540-7041 \\ Seon Ho Kim \\ https://orcid.org/0000-0002-9350-1853 \\ Sung Sill Lee \\ https://orcid.org/0000-0002-4621-4333
}

Submitted Mar 31, 2020; Revised Apr 24, 2020; Accepted Jun 5, 2020
Objective: The metabolites that constitute the rumen fluid and milk in dairy cattle were analyzed using proton nuclear magnetic resonance $\left({ }^{1} \mathrm{H}-\mathrm{NMR}\right)$ spectroscopy and compared with the results obtain for other dairy cattle herds worldwide. The aim was to provide basic dataset for facilitating research on metabolites in rumen fluid and milk.

Methods: Six dairy cattle were used in this study. Rumen fluid was collected using a stomach tube, and milk was collected using a pipeline milking system. The metabolites were determined by ${ }^{1} \mathrm{H}-\mathrm{NMR}$ spectroscopy, and the obtained data were statistically analyzed by principal component analysis, partial least squares discriminant analysis, variable importance in projection scores, and metabolic pathway data using Metaboanalyst 4.0.

Results: The total numbers of metabolites in rumen fluid and milk were measured to be 186 and 184, and quantified as 72 and 109, respectively. Organic acid and carbohydrate metabolites exhibited the highest concentrations in rumen fluid and milk, respectively. Some metabolites that have been associated with metabolic diseases (acidosis and ketosis) in cows were identified in rumen fluid, and metabolites associated with ketosis, somatic cell production, and coagulation properties were identified in milk.

Conclusion: The metabolites measured in rumen fluid and milk could potentially be used to detect metabolic diseases and evaluate milk quality. The results could also be useful for metabolomic research on the biofluids of ruminants in Korea, while facilitating their metabolic research.

Keywords: Dairy Cattle; Metabolites; Milk; Rumen Fluid; ${ }^{1} \mathrm{H}-\mathrm{NMR}$ Spectroscopy

\section{INTRODUCTION}

Metabolomic studies have been actively conducted to not only investigate metabolic diseases found in animals, but also related human diseases, drug toxicity, and altered gene functions [1-3]. Metabolomics is the study of metabolic mechanisms that are achieved by measuring metabolic products in biological specimens as a whole and by elucidating the underlying chemical processes [2]. The most common techniques used for metabolomics are nuclear magnetic resonance (NMR) spectroscopy, liquid chromatography-mass spectrometry (LC/MS) and gas chromatography-MS (GC/MS). NMR spectroscopy is less sensitive than LC/MS and GC/MS, while providing at relatively simple sample preparation and highly reproducible molecule quantification [3,4]. In addition, NMR is a powerful analytical technique for the identification of metabolic biomarkers [5]. Therefore, NMR would be suitable for the analysis of numerous metabolites in the biofluids of ruminants.

Numerous researches have previously analyzed the metabolites in ruminant biofluids (e.g., rumen fluid, serum, milk, and urine) using NMR spectroscopy, and by the identification and quantification of metabolites through concentration comparison [1,6-8]. 
Moreover, metabolic pathways have been statistically analyzed by principal component analysis (PCA), partial least square discriminant analysis (PLS-DA), determination of variable importance in projection (VIP) scores, and hierarchical clustering analysis (with heatmaps) [1,6-8]. In particular, research is being conducted on finding new biomarkers for metabolic diseases found in ruminants (acidosis, ketosis, etc.) as well as for the early diagnosis of these diseases that may occur in the livestock industry; this has been achieved by identifying specific metabolites in ruminant biofluids $[9,10]$. Therefore, metabolites in ruminant biofluids must be studied to prevent and minimize the damages caused by metabolic diseases.

In Korea, NMR metabolomics studies have been conducted on samples such as natural food (potatoes and Astragalus root) products [11,12], human urine [13], liver, serum, urine of piglets [14], and obese mice [15]. Recently, metabolomic research has been conducted by comparing the composition of rumen fluid in Hanwoo's cattle with those of the volatile fatty acids and monosaccharides in metabolites by using NMR spectroscopy, high performance liquid chromatography and high-performance anion-exchange chromatography [16]. A study has also investigated the effects of different roughage diets that were fed at concentrated ratios on the milk productivity and milk metabolites in dairy cows [17]. However, extremely few studies have been conducted on metabolites that constitute ruminant biofluids in Korea by using proton nuclear magnetic resonance ( $\left.{ }^{1} \mathrm{H}-\mathrm{NMR}\right)$.

Therefore, this study aimed to measure the metabolites in dairy cow rumen fluid and milk by using ${ }^{1} \mathrm{H}-\mathrm{NMR}$ spectroscopy; these metabolites were quantified and classified to construct a database for each sample, wherein the concentration of each metabolite was provided. The role of metabolites in rumen fluid and milk observed in this study were compared to that of the metabolites in ruminant biofluids reported in a previous study. Moreover, the results of this study provide a useful a database for the analysis of metabolites in ruminant biofluids.

\section{MATERIALS AND METHODS}

All experimental protocols used in this study were approved by the National Institute of Animal Science Department of Animal Resources Development Dairy Science Division (Cheonan, Chungcheongnam-do, Korea; NIAS-201908).

\section{Animals and sample collection}

Six dairy cattle were used in this study, and all animals were fed the same diet that was composed of total mixed ration (TMR); the fed amounts were defined by their voluntary intake. The chemical composition of the TMR have been presented in Table 1. Rumen fluid samples were collected
Table 1. Ingredients and nutrients of the experimental diets

\begin{tabular}{|c|c|}
\hline Items & Value (\% of dry matter) \\
\hline \multicolumn{2}{|l|}{ Ingredients } \\
\hline Concentrate & 15.3 \\
\hline Soybean meal & 2.40 \\
\hline Corn silage & 47.2 \\
\hline Alfalfa hay & 7.10 \\
\hline Tall fescue & 9.40 \\
\hline Timothy & 5.90 \\
\hline Energy booster ${ }^{1)}$ & 7.10 \\
\hline Cash gold ${ }^{1)}$ & 4.50 \\
\hline Lyzin-plus ${ }^{2)}$ & 0.20 \\
\hline Limestone & 0.20 \\
\hline Zin care ${ }^{1)}$ & 0.10 \\
\hline Supex- $F^{1)}$ & 0.50 \\
\hline Trace minerals ${ }^{3)}$ & 0.05 \\
\hline Vitamins premix ${ }^{4)}$ & 0.05 \\
\hline \multicolumn{2}{|l|}{ Chemical composition } \\
\hline Dry matter (\%) & 53.2 \\
\hline Crude protein & 10.0 \\
\hline Neutral detergent fiber & 28.2 \\
\hline Acid detergent fiber & 16.9 \\
\hline Calcium & 0.40 \\
\hline Phosphorus & 0.15 \\
\hline \multicolumn{2}{|c|}{$\begin{array}{l}\text { 1) Cofavet, Cheonan, Korea. } \\
\text { 2) A.N.Tech, Cheonan, Korea. } \\
\text { 3) Trace minerals, contained } 0.40 \% \mathrm{Mg}, 0.20 \% \mathrm{~K}, 4.00 \% \mathrm{~S}, 0.08 \% \mathrm{Na} \text {, } \\
0.03 \% \mathrm{Cl}, 400 \mathrm{mg} \text { of Fe/kg, } 60,042 \mathrm{mg} \text { of } \mathrm{Zn} / \mathrm{kg}, 16,125 \mathrm{mg} \text { of } \mathrm{Cu} / \mathrm{kg} \text {, and } \\
42,375 \mathrm{mg} \text { of } \mathrm{Mn} / \mathrm{kg} \text {. } \\
\text { 4) Vitamins premix, provided approximately } 5,000 \mathrm{KIU} \text { of vitamin } \mathrm{A} / \mathrm{kg} \text {, } \\
1,000 \mathrm{KIU} \text { of vitamin D/kg, } 33,500 \mathrm{mg} \text { of vitamin } \mathrm{E} / \mathrm{kg} \text {, and } 2,400 \mathrm{mg} \text { of } \\
\text { vitamin C/kg. }\end{array}$} \\
\hline
\end{tabular}

using stomach tube from dairy cows. All rumen fluid collected using conical tube ( $30 \mathrm{~mL}$ each). Subsequently, rumen fluid samples centrifuged at $806 \mathrm{~g}$ for $15 \mathrm{~min}$ to remove feed particles and the supernatant was stored at $-80^{\circ} \mathrm{C}$ until analyzed for metabolites using ${ }^{1} \mathrm{H}-\mathrm{NMR}$. Milk samples were collected by using pipeline milking system and then transferred to conical tubes $(30 \mathrm{~mL}$ each). Samples were stored at $-80^{\circ} \mathrm{C}$ until analyzing for metabolites by ${ }^{1} \mathrm{H}-\mathrm{NMR}$.

\section{NMR spectroscopy}

The rumen fluid sample was recentrifuged at $12,902 \mathrm{~g}$ for 10 min and the supernatant was collected $300 \mu \mathrm{L}$. Standard buffer solution (2,2,3,3-d(4)-3-(trimethylsilyl)propionic acid [TSP] sodium salt) was added to $300 \mu \mathrm{L}$ of supernatant in deuterium oxide $\left(\mathrm{D}_{2} \mathrm{O}\right)$ solvent/standard buffer solution (300 $\mu \mathrm{L})$. The supernatants $(600 \mu \mathrm{L})$ were transferred to $5 \mathrm{~mm}$ NMR tubes for NMR analysis [6].

The collected milk sample was centrifuged at 4,000 g for $15 \mathrm{~min}$ to remove the lipid layer in supernatant. Thereafter, the mixture of milk $(250 \mu \mathrm{L})$ and $\mathrm{D}_{2} \mathrm{O}(300 \mu \mathrm{L})$ were transferred to $5 \mathrm{~mm}$ NMR tubes for NMR analysis [6].

${ }^{1} \mathrm{H}-\mathrm{NMR}$ spectra of rumen fluid and milk samples were 
obtained on a SPE-800 MHz NMR-MS Spectrometer (Bruker BioSpin AG, Billerica, MA, USA) at $64 \mathrm{~K}$ using a $5 \mathrm{~mm}$ tripleresonance inverse cryoprobe with Z-gradients (Bruker BioSpin CO., USA). The pulse sequence used for the rumen fluid and milk were a NOESY and Carr-Purcell-Meiboom-Gill pulse sequence collecting 64,000 data points with 128 transients, a spectral width of $16,025.641 \mathrm{~Hz}$, a relaxation delay of 4.0 $\mathrm{s}$, and an acquisition time of $2.0 \mathrm{~s}$.

\section{NMR metabolite measurement, quantification, and} statistical analysis

The processed spectra were imported into the Chenomx NMR suite 8.4 software (Chenomx, Edmonton, Canada) for identification and quantification. The baseline and phase were matched for comparison between samples using the NMR processor. The following procedure was employed for qualitative and quantitative analysis of the metabolites in samples: the spectral width was $10 \mathrm{ppm}$ and was referenced to the TSP signal at $0 \mathrm{ppm}$. The resources used were the Livestock Metabolite Database (http://www.lmdb.ca), Bovine Metabolite Database (http://www.bmdb.ca), and Chenomx library. Moreover, metabolite qualitative and quantitative were performed using the Chenomx profiler program.

Statistical analyses of the metabolite data were conducted using MetaboAnalyst version 4.0 (http://www.metaboanalyst. ca), an open source R-based program for metabolomics. During the statistical analysis the resulting metabolites were subjected to sample normalization, data transformation, and data scaling by using the "sum", "log", "pareto" functions, respectively. Univariate Student's t-tests were used to identify differences between the metabolite profiles of the rumen fluid and milk samples. PCA and PLS-DA were used as multivariate data analysis techniques to generate a classification model and provide quantitative information for discriminating the metabolites. The different rumen fluid and milk metabolites were determined on the basis of a statistically significant threshold of VIP scores. Metabolites with VIP scores higher than 1.5 were obtained through PLS-DA. Metabolic pathways were quantified and, common metabolites in rumen fluid as well as milk metabolites of the other studied animals were statistically analyzed by MetaboAnalyst 4.0 for performing a metabolic pathway analysis, based on a database source by Kyoto encyclopedia of genes and genomes (http://www. kegg.com).

\section{RESULTS}

\section{Measured quantification of metabolites by ${ }^{1} \mathrm{H}-\mathrm{NMR}$ spectroscopy}

The results in shown Figure 1 and Supplementary Table S1, $S 2$, and S3 reveal the measured and quantified compound in rumen fluid (A) and milk (B) that were measured by ${ }^{1} \mathrm{H}$ NMR. In the rumen fluid, 186 metabolites were measured and classified into 13 chemical classes. The classes with the most metabolites were others (34), carboxylic acids (27), and carbohydrates (27); the classes with the three highest concentrations were organic acids $(22.759 \mathrm{mM})$, carbohydrates $(4.871 \mathrm{mM})$ and lipids $(0.961 \mathrm{mM})$. In addition, 72 metabolites were quantified $(\mathrm{n} \geq 4)$ in the rumen fluid. In milk, 184 metabolites were measured and classified into 14 chemical

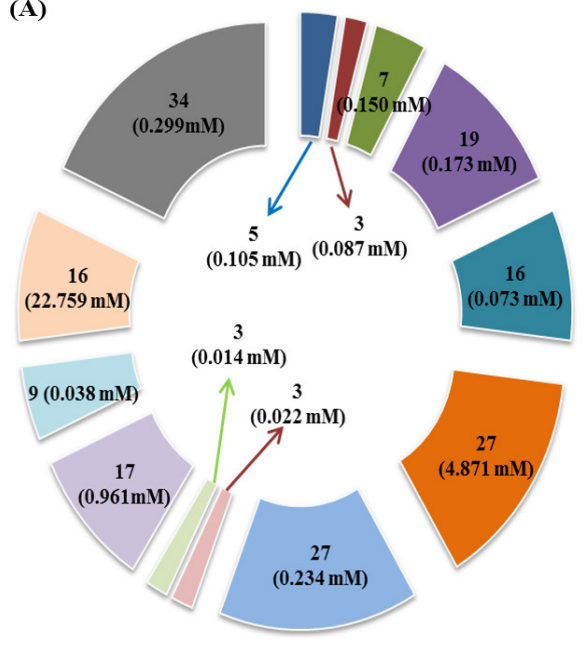

(B)
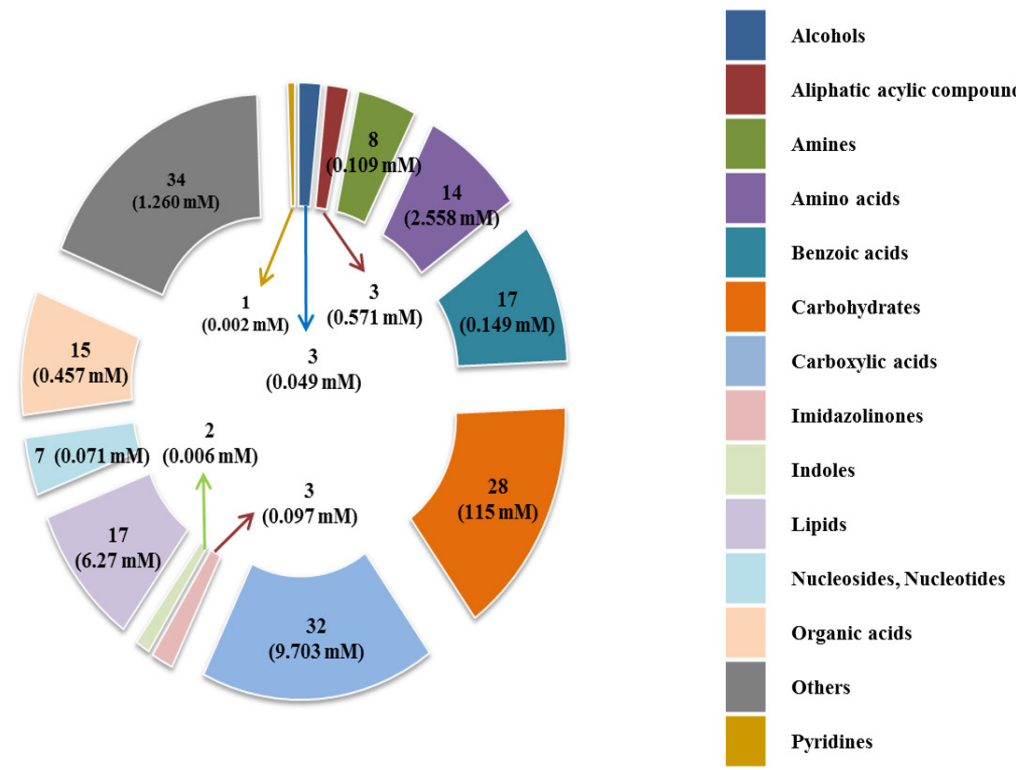

Figure 1. The classification of measured metabolites according to chemical class in rumen fluid (A) and, milk (B) by ${ }^{1} \mathrm{H}-\mathrm{NMR}$ analysis. Each square box color indicates the classification of metabolites, the numbers represents the measured metabolites, and the numbers in parentheses indicate the sum of the total concentrations of the measured metabolites. ${ }^{1} \mathrm{H}-\mathrm{NMR}$, proton nuclear magnetic resonance. 
classes. The classes with the most metabolites were others (34), carboxylic acids (32), and carbohydrates (28); the classes with the three highest concentrations were carbohydrates (115 mM), carboxylic acids (9.703 mM), and lipids (6.270 $\mathrm{mM})$. In addition, 109 metabolites were quantified $(\mathrm{n} \geq 4)$ in milk.

\section{${ }^{1} \mathrm{H}$-NMR spectroscopy analysis}

Representative ${ }^{1} \mathrm{H}$-NMR spectra of 58 and 63 metabolites measured as single, doublet, and triplet peaks, as well as the reference substance TSP, in the rumen fluid and milk samples are shown in Supplementary Figures S1 and S2, respectively. Our data showed that the metabolites were different between rumen fluid and milk. To visualize the differences among the metabolites data, we performed PCA and PLS-DA (Figures $2,3)$. Both score plots revealed differences in rumen fluid and milk, which were well separated in PC1 (39.8\%) and PC2 (9.2\%) for PCA and PC1 (39.8\%) and PC2 (7.3\%) PLS-DA. These results indicated significant variation among the different classes and concentrations of metabolites in the rumen fluid and milk. As shown in Figure 4, the two biofluids exhibited completely different metabolite profiles. VIP scores were also utilized to identify the metabolites that affected the differentiation in the PLS-DA score plot, and 21 metabolites were found to be significantly different (VIP score $>1.5$ ) in the rumen fluid and milk (Figure 4). In the rumen fluid, 14 metabolites (propionate, butyrate, valerate, acetate, caprate, isovalerate, glucose, isobutyrate, $\mathrm{N}$-phenylacetylglycine, acetamide, 3-phenylpropionate, 4-methylhistidine, $\mathrm{N}$-acetylglycine, and 2-aminobutyrate) had significantly higher concentrations than those present in milk. In milk, 7 metabolites (guanidinoacetate, glycine, lactose, glycolate, tartrate, galactose, and sn-glycero-3-phosphocholine) had significantly higher concentrations than those present in the rumen fluid.

\section{Top 30 average concentrations of rumen fluid and milk} metabolites

The top 30 average concentrations of metabolites in the rumen fluid and milk are shown in Table 2 and 3. Among the metabolites measured in the rumen fluid, acetate, propionate, and butyrate (classified as organic acids) had the highest concentrations. In contrast, uracil (classified as a nucleoside and nucleotide), 3-hydroxyphenylacetate (classified as a carboxylic acid), and imidazole (classified as an imidazolinone) had the lowest concentrations. Among the metabolites measured in milk, lactose (classified as a carbohydrate), guanidinoacetate (classified as a carboxylic acid), and ethylene glycol (classified as a lipid) had the highest concentrations. In contrast, tartrate (classified as a benzoic acid), $\mathrm{N}$-carbamoyl- $\beta$-alanine (classi-

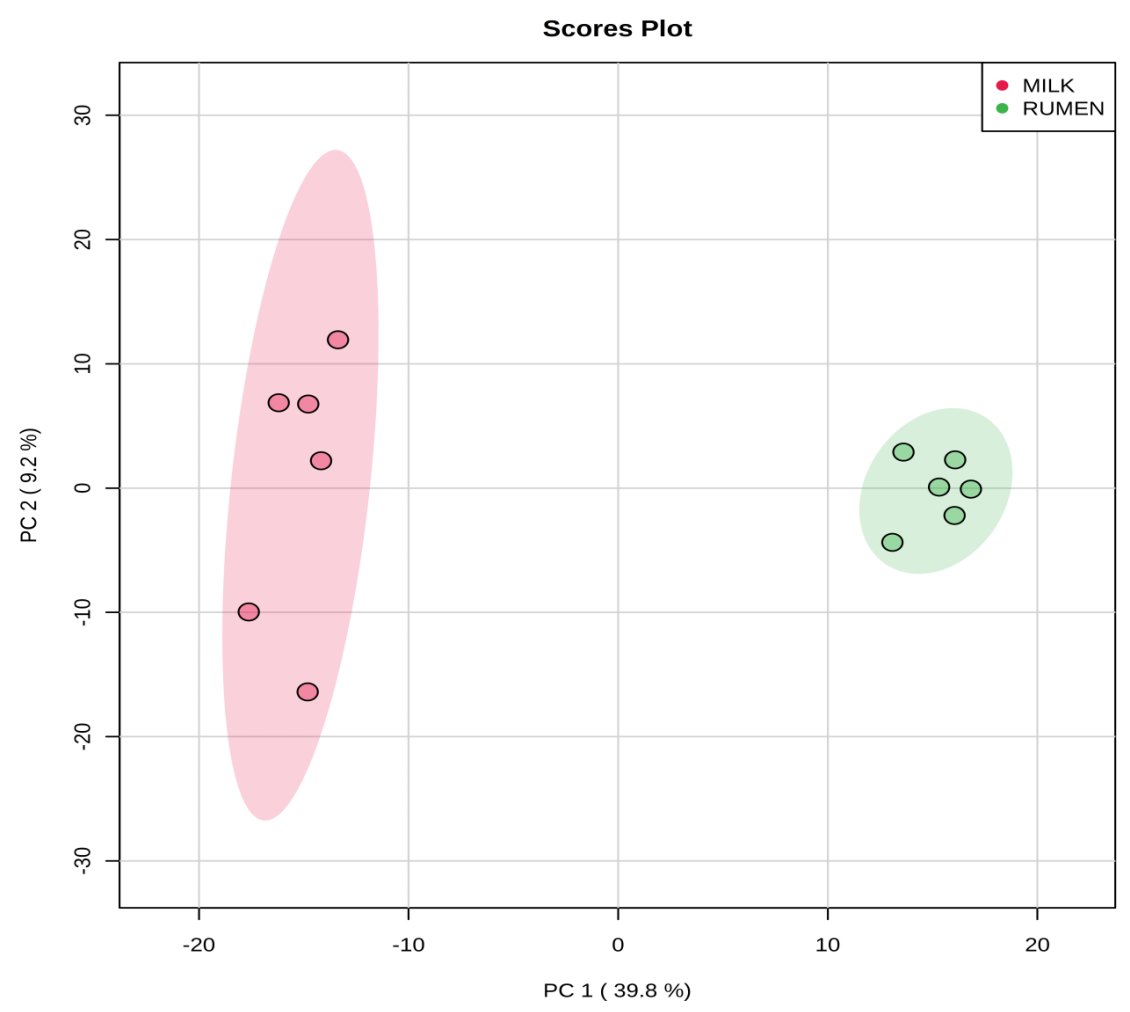

Figure 2. Principal components analysis (PCA) score plot based on metabolites data in rumen fluid and milk by ${ }^{1} \mathrm{H}-\mathrm{NMR}$ analysis. On the score plot, each point represents an individual sample, with the green dot representing the rumen fluid group $(n=6)$, and the red dot representing the milk group $(n=6)$. The abscissa and ordinate represent the variance associated with PC 1 and 2 , respectively. ${ }^{1} \mathrm{H}-\mathrm{NMR}$, proton nuclear magnetic resonance. 


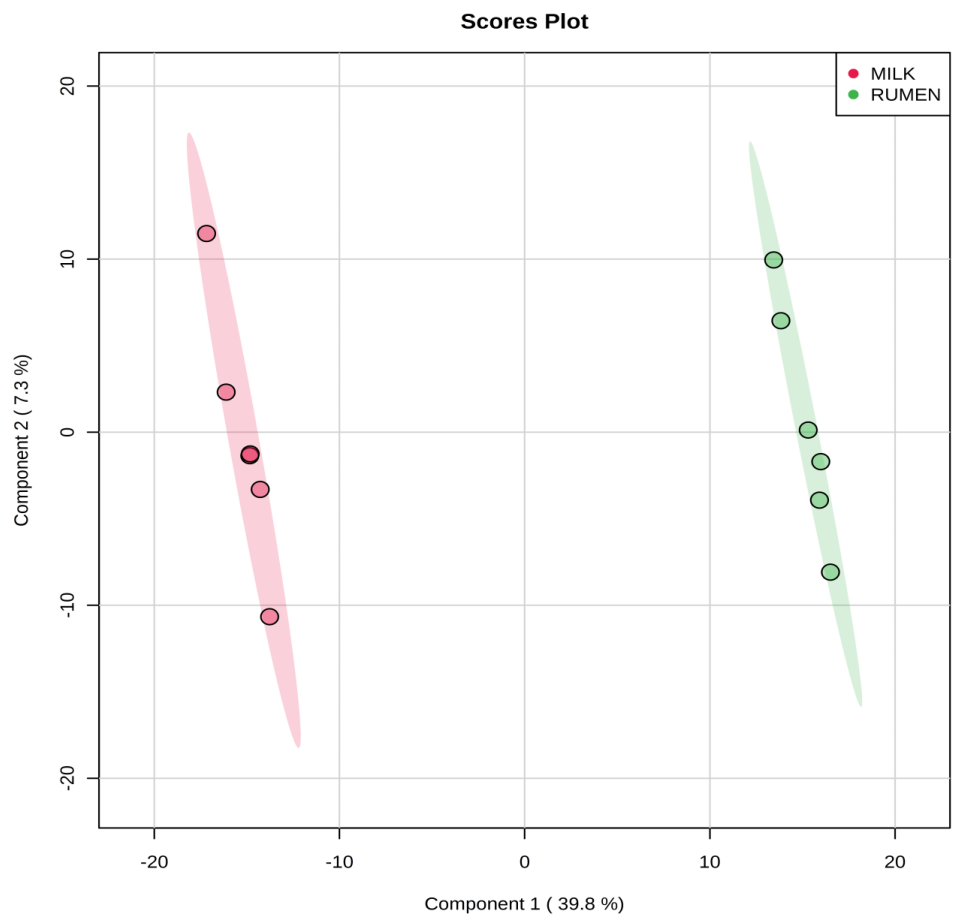

Figure 3. Partial least square discriminant analysis (PLS-DA) score plot of rumen fluid and milk, by ${ }^{1} H$-NMR analysis. The shaded ellipses represent the $95 \%$ confidence interval estimated from the score. On the score plot, each point represents an individual sample, with the green dot representing the rumen fluid group $(n=6)$, and the red dot representing the milk group $(n=6)$. The abscissa and ordinate represent the variance associated with components 1 and 2 , respectively. ${ }^{1} \mathrm{H}-\mathrm{NMR}$, proton nuclear magnetic resonance.

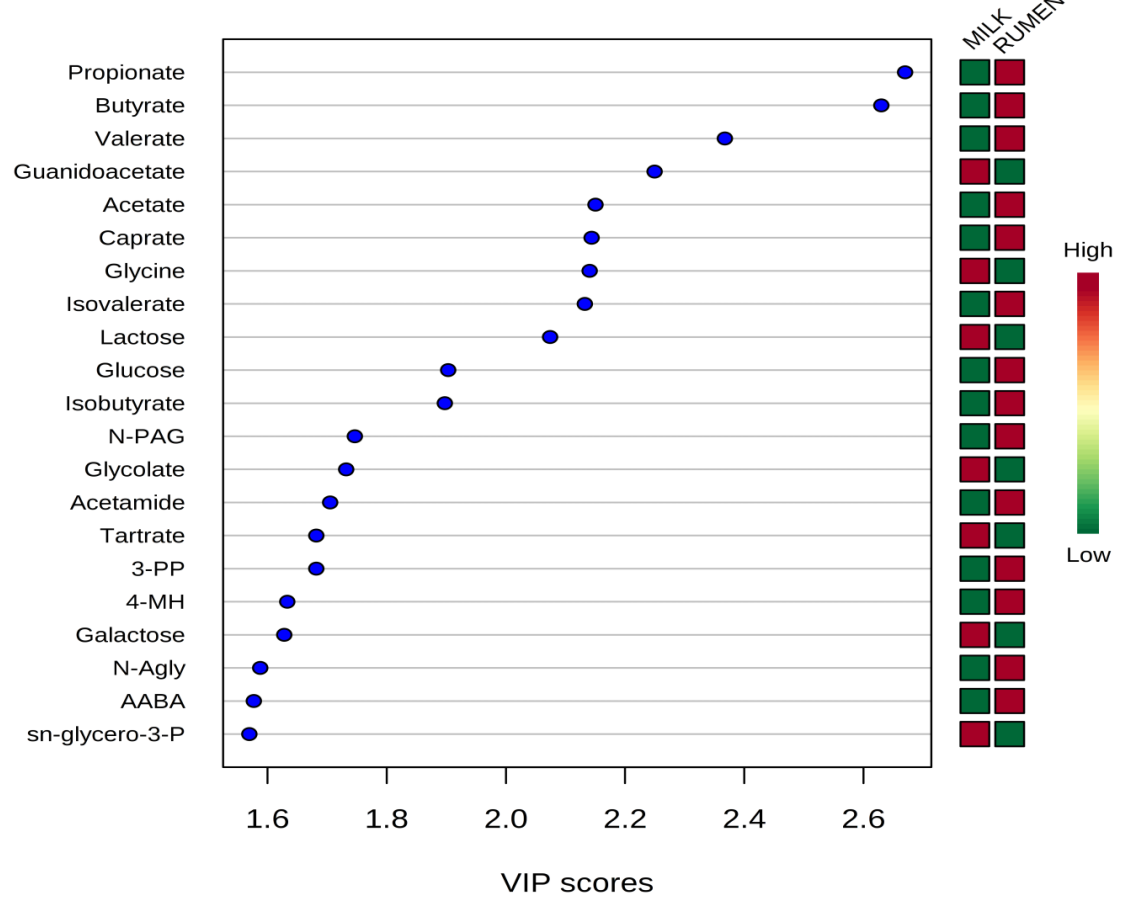

Figure 4. Variable importance in projection (VIP) scores of metabolites in rumen fluid and milk by ${ }^{1} \mathrm{H}-\mathrm{NMR}$ analysis. The selected metabolites were those with VIP score>1.5. Heat map with red or green boxes on the right indicates high and low abundance ratio, respectively, of the corresponding metabolite in rumen fluid and milk. VIP score was based on the PLS-DA model. 'H-NMR, proton nuclear magnetic resonance; PLS-DA, partial least square discriminant analysis; N-PAG, N-phenylacetylglycine; 3-PP, 3-phenylpropionate; 4-MH, 4-methylhistidine; N-agly, N-acetylglycine; AABA, 2-aminobutyrate; sn-glycero-3-P, sn-glycero-3-phosphocholine. VIP score value: propionate, 2.6698; butyrate, 2.6299; valerate, 2.3674; guanidoacetate, 2.2495; acetate, 2.1503; caprate, 2.1438; glycine, 2.1405; isovalerate, 2.1325; lactose, 2.0742; glucose, 1.9032; isobutyrate, 1.8975; N-PAG, 1.7465; glycolate, 1.7320; acetamide, 1.7051; tartrate, 1.6819; 3-PP, 1.6819; 4-MH, 1.6332; galactose, 1.6281; N-Agly, 1.5878; AABA, 1.5771; sn-glycero-3-P, 1.5696. 
Table 2. Average concentrations (mean \pm standard deviation) of top 30 metabolites in rumen fluid by ${ }^{1} H-N M R$ analysis ( $\left.n \geq 4\right)$

\begin{tabular}{|c|c|c|}
\hline Metabolites & Classification & Concentration $(\mu \mathrm{M})$ \\
\hline Acetate & Organic acids & $14,474.47 \pm 1,900.36$ \\
\hline Propionate & Organic acids & $4,434.27 \pm 716.46$ \\
\hline Butyrate & Organic acids & $3,097.57 \pm 566.60$ \\
\hline Valerate & Organic acids & $330.00 \pm 61.46$ \\
\hline Isobutyrate & Organic acids & $165.76 \pm 23.15$ \\
\hline Acetamide & Organic acids & $118.95 \pm 19.22$ \\
\hline Methylamine & Amines & $115.93 \pm 22.24$ \\
\hline 3-Phenylpropionate & Others & $108.53 \pm 19.83$ \\
\hline Isovalerate & Organic acids & $71.12 \pm 44.13$ \\
\hline Glucose & Carbohydrates & $65.08 \pm 22.81$ \\
\hline Caprate & Lipids & $60.98 \pm 19.87$ \\
\hline Isopropanol & Alcohols & $42.88 \pm 52.55$ \\
\hline Ribose & Carbohydrates & $36.33 \pm 15.15$ \\
\hline 2-Aminobutyrate & Amino acids & $27.95 \pm 10.88$ \\
\hline Alanine & Amino acids & $26.75 \pm 14.31$ \\
\hline Phenylacetate & Organic acids & $22.53 \pm 12.09$ \\
\hline Maltose & Carbohydrates & $22.15 \pm 14.79$ \\
\hline Glucose-6-phosphate & Carbohydrates & $21.18 \pm 23.44$ \\
\hline Alloisoleucine & Carboxylic acids & $19.98 \pm 12.61$ \\
\hline $\mathrm{N}$-acetylglucosamine & Carbohydrates & $19.07 \pm 17.91$ \\
\hline 3-Hydroxy-3-methylglutarate & Lipids & $16.45 \pm 7.26$ \\
\hline N-acetylglycine & Carboxylic acids & $16.23 \pm 7.02$ \\
\hline Xylitol & Carbohydrates & $15.75 \pm 14.96$ \\
\hline Biotin & Others & $14.58 \pm 13.32$ \\
\hline Lactose & Carbohydrates & $11.23 \pm 6.37$ \\
\hline Fructose & Carbohydrates & $10.40 \pm 7.98$ \\
\hline Erythritol & Carbohydrates & $9.50 \pm 6.46$ \\
\hline Uracil & Nucleosides, nucleotides & $9.25 \pm 4.26$ \\
\hline 3-Hydroxyphenylacetate & Carboxylic acids & $9.20 \pm 9.31$ \\
\hline Imidazole & Imidazolinones & $9.16 \pm 2.55$ \\
\hline
\end{tabular}

${ }^{1} \mathrm{H}-\mathrm{NMR}$, proton nuclear magnetic resonance.

fied as an organic acid), and acetone (classified as a others compound) had the lowest concentrations.

\section{Common metabolites and metabolic pathways in the rumen fluid and milk}

Fourth-three common metabolites were quantified $(n \geq 4)$ in the rumen fluid and milk (Supplementary Table S1-3). An analysis showed that the common metabolites (Table 4, Figure 5) followed 16 metabolic pathways, among which the top five pathways were pyruvate metabolism, glycolysis/gluconeogenesis, glyoxylate and dicarboxylate metabolism, galactose metabolism, and glycerophospholipid metabolism. The metabolites in the relevant pathways were mainly carbohydrates and amino acids.

\section{DISCUSSION}

Metabolites in ruminant biofluids can be affected by the species of ruminant, type of feed consumed, breeding environment and season, and the adopted analytical technique. Saleem et al [18] analyzed a total of 246 metabolites using
${ }^{1} \mathrm{H}-\mathrm{NMR}$, GC/MS, LCMS/MS, and ICP-MS to study bovine rumination according to feeding concentrate ratio, 50 of which were measured by $1 \mathrm{H}-\mathrm{NMR}$. In this study, a total of 186 metabolites were measured by ${ }^{1} \mathrm{H}-\mathrm{NMR}, 72$ of which were quantified in rumen fluid. O'Callaghan et al [6] studied bovine milk under different types of irradiation type by ${ }^{1} \mathrm{H}$ NMR and quantified a total of 49 metabolites. In this study, a total of 184 metabolites were measured by ${ }^{1} \mathrm{H}-\mathrm{NMR}$, among which 109 were quantified in milk. It is possible that the higher NMR frequency and the Chenomx programs used in this study resulted in the quantification of more metabolites.

PCA is a multivariate method that can be used to classify and analyze large amounts of data with numerous variables and provide information on metabolite changes [19]. PLS-DA visualizes metabolic changes that are likely to affect classification for two or more individuals. VIP scores and heatmaps indicate the differences in the contributions of metabolites when the two groups are divided according to PLS-DA [20]. In Korea, multivariate analysis has been widely used for food origin determination $[11,12]$ and human diseases [13]. Multivariate analysis is also an essential method for metabolite 
Table 3. Average concentrations (mean \pm standard deviation) of top 30 metabolites in milk by ${ }^{1} H-N M R$ analysis $(n \geq 4)$

\begin{tabular}{|c|c|c|}
\hline Metabolites & Classification & Concentration $(\mu \mathrm{M})$ \\
\hline Lactose & Carbohydrates & $72,183.65 \pm 12,418.97$ \\
\hline Guanidoacetate & Carboxylic acids & $8,620.95 \pm 2,606.36$ \\
\hline Ethylene glycol & Lipids & $3,860.08 \pm 859.05$ \\
\hline 1,3-Dihydroxyacetone & Carbohydrates & $2,953.88 \pm 2,719.11$ \\
\hline Glycine & Amino acids & $2,154.20 \pm 722.28$ \\
\hline Glycolate & Lipids & $1,701.72 \pm 984.03$ \\
\hline sn-Glycero-3-phosphocholine & Others & $267.63 \pm 110.73$ \\
\hline Arabinose & Others & $263.78 \pm 362.78$ \\
\hline Ribose & Carbohydrates & $240.95 \pm 160.76$ \\
\hline Isocitrate & Carbohydrates & $226.58 \pm 157.30$ \\
\hline Fructose & Carbohydrates & $217.50 \pm 72.93$ \\
\hline Lactulose & Carbohydrates & $165.84 \pm 61.39$ \\
\hline Choline & Lipids & $150.60 \pm 140.69$ \\
\hline Gluconate & Organic acids & $121.65 \pm 80.61$ \\
\hline Mannose & Carbohydrates & $106.08 \pm 81.75$ \\
\hline O-Phosphocholine & Aliphatic acylic compounds & $102.15 \pm 142.06$ \\
\hline Cellobiose & Others & $100.14 \pm 40.66$ \\
\hline Xylose & Carbohydrates & $99.42 \pm 61.97$ \\
\hline Trimethylamine $\mathrm{N}$-oxide & Aliphatic acylic compounds & $98.88 \pm 203.24$ \\
\hline Fucose & Others & $94.64 \pm 48.42$ \\
\hline O-acetylcarnitine & Lipids & $83.63 \pm 48.79$ \\
\hline Acetone & Others & $80.68 \pm 79.94$ \\
\hline
\end{tabular}

${ }^{1} \mathrm{H}-\mathrm{NMR}$, proton nuclear magnetic resonance.

Table 4. Pathway analysis with common quantified $(n \geq 4)$ metabolites in rumen fluid and milk

\begin{tabular}{|c|c|c|c|c|c|c|}
\hline Metabolic pathway & Total Cmpd ${ }^{1)}$ & Hits $^{2)}$ & p-value & -Log (p-value) & $\mathrm{FDR}^{3)}$ & Impact $^{4)}$ \\
\hline Pyruvate metabolism & 22 & 1 & $3.42 \times 10^{-11}$ & 24.10 & $3.42 \times 10^{-10}$ & 0.06 \\
\hline Glycolysis/gluconeogenesis & 26 & 1 & $3.42 \times 10^{-11}$ & 24.10 & $3.42 \times 10^{-10}$ & 0.03 \\
\hline Galactose metabolism & 27 & 2 & $1.20 \times 10^{-8}$ & 18.24 & $9.01 \times 10^{-8}$ & 0.15 \\
\hline Glycerophospholipid metabolism & 26 & 3 & $2.50 \times 10^{-5}$ & 10.60 & $1.50 \times 10^{-4}$ & 0.08 \\
\hline Ether lipid metabolism & 20 & 1 & $5.47 \times 10^{-5}$ & 9.81 & $2.73 \times 10^{-4}$ & 0.00 \\
\hline Tryptophan metabolism & 41 & 2 & $6.14 \times 10^{-4}$ & 7.40 & $2.30 \times 10^{-3}$ & 0.05 \\
\hline Glycine, serine and threonine metabolism & 34 & 3 & $7.44 \times 10^{-4}$ & 7.20 & $2.42 \times 10^{-3}$ & 0.13 \\
\hline Vitamin B6 metabolism & 9 & 2 & $8.06 \times 10^{-4}$ & 7.12 & $2.42 \times 10^{-3}$ & 0.08 \\
\hline Arginine and proline metabolism & 38 & 1 & $1.52 \times 10^{-3}$ & 6.49 & $4.15 \times 10^{-3}$ & 0.00 \\
\hline Amino sugar and nucleotide sugar metabolism & 37 & 2 & $5.26 \times 10^{-3}$ & 5.25 & $1.32 \times 10^{-2}$ & 0.07 \\
\hline
\end{tabular}

\footnotetext{
1) Total Cmpd, the total number of compounds in the pathway.

2) Hit, the actually matched number from the user uploaded data.

3) FDR, the p-value adjusted using false discovery rate.

${ }^{4)}$ Impact, the pathway impact value calculated from pathway topology analysis.
} 


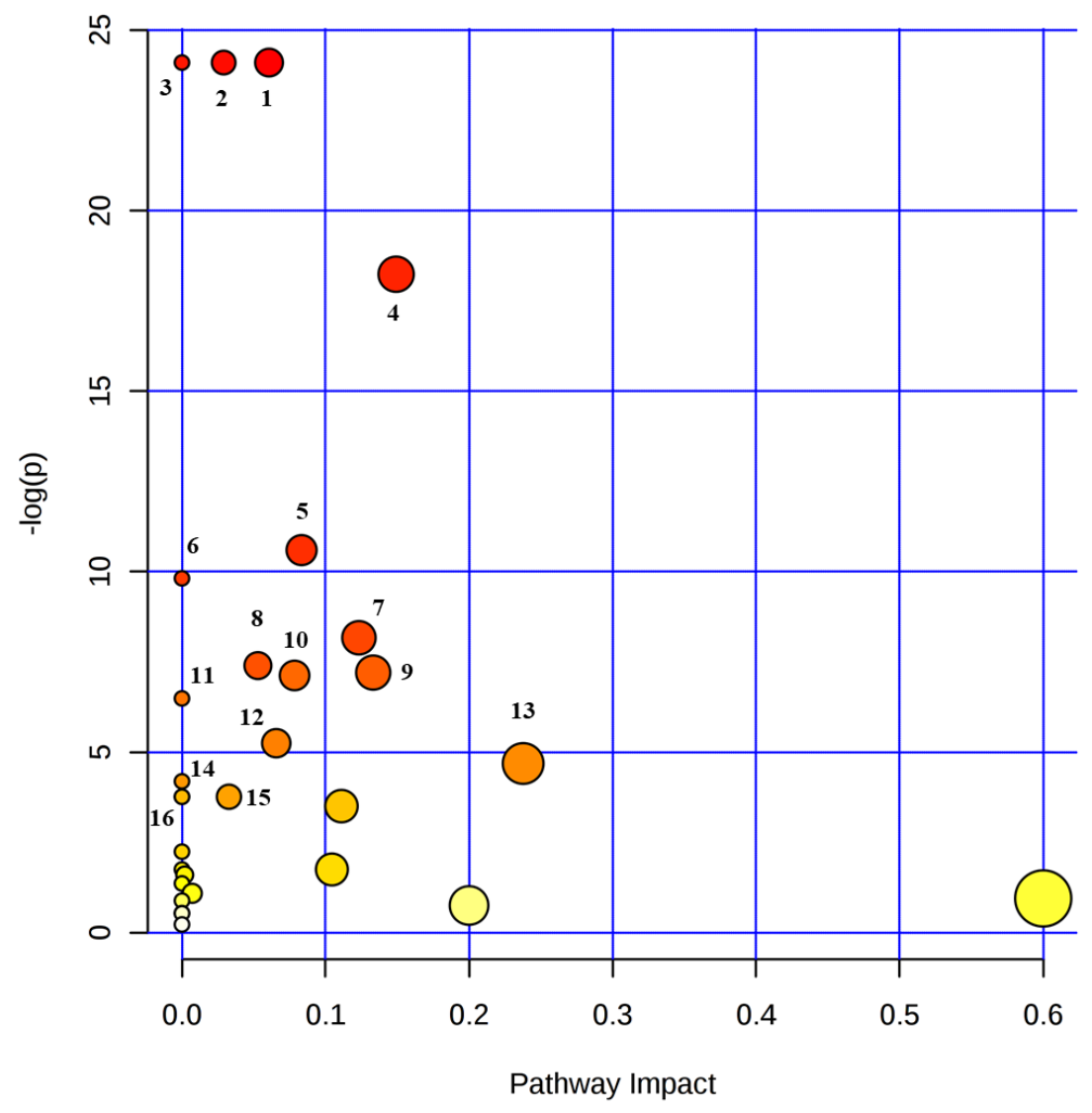

Figure 5. Metabolic pathway mapping of common quantified $(n \geq 4)$ metabolites between rumen fluid and milk. The pathway impact analysis was performed using Metaboanalyst 4.0 software. The $x$-axis represents the pathway impact, and $y$-axis represents the pathway enrichment. The results are presented graphically as a bubble plot. The darker color and larger the size represent higher $\mathrm{p}$-value from enrichment analysis and greater impact from the pathway topology analysis, respectively. Metabolic pathway name: 1, pyruvate metabolism; 2, glycolysis/glucoconeogenesis; 3 , glyoxylate and dicarboxylate metabolism; 4, galactose metabolism; 5, glycerophospholipid metabolism; 6, ether lipid metabolism; 7, starch and sucrose metabolism; 8, tryptophan metabolism; 9, glycine, serine and threonine metabolism; 10, vitamin B $_{6}$ metabolism; 11, arginine and proline metabolism; 12, amino sugar and nucleotide sugar metabolism; 13, histidine metabolism; 14, phenylalanine metabolism; 15, citrate cycle (tricarboxylic acid cycle); 16, propanoate metabolism.

research; thus, studies on the metabolites found in ruminant biofluids are also be necessary. The PCA of the metabolites in rumen fluid and milk showed a separation between the groups. The PLS-DA was influenced by propionate, butyrate, valerate in rumen fluid and guanidinoacetate, glycine, and lactose metabolites in milk, which also demonstrated a separation between the groups.

Previous studies have shown that acetate, propionate, and butyrate in the rumen account for approximately $70 \%$ of ruminant energy and the majority of metabolite concentrations [18], which in accordance with this study. In terms of metabolic diseases, a higher concentrate ratio of the agricultural feed can cause acidosis [21]. According to Ametaj et al [22], a diet with a high concentrate ratio increases methylamine, glucose, alanine, maltose, propionate, uracil, valerate, xanthine, ethanol, and phenylacetate concentrations and decreases 3-phenylpropionate concentrations. In this study, the feeding concentrate ratio was measured in relation to the metabo- lites. As reported by Wang et al [23], the concentrations of metabolites based on biogenic amines (tyramine, putrescine, histamine, methylamine, and tryptamine) in the rumen fluid were observed to be higher in bovines suffering from acidosis. Sato and Shiogama [24] reported that changes in the concentrations of acetone and isopropanol in rumen fluid might be an indicator of ketosis. Compared with the metabolites detected in the above-mentioned previous studies [22-24], a majority of these were also detected in the rumen fluid in this study, including the diuretic-related biogenic amine metabolite histamine, methylamine, acetone, and isopropanol. Therefore, the metabolites measured in rumen fluid in this study can be used to verify the occurrence of diseases such as acidosis and ketosis in dairy cattle.

Numerous studies have been conducted on metabolites present in milk. Among them, a few have determined the chemical composition and nutritional value of milk along with the bioactive compounds present in milk; further, they 
have elucidated, the potential use of biomarkers in milk as a diagnostic tool using NMR spectroscopy [25]. According to Klein et al [9], the milk samples from cows exhibiting ketosis showed higher concentrations of ketone body metabolites (3-hydroxybutyrate [BHBA], acetoacetate, and acetone) and a higher glycerophosphocholine to phosphocholine ratio. Moreover, the presence of somatic cells was found to affect milk quality. Sundekilde et al [25] classified BHBA, lactate, lactose, hippurate, acetate, butyrate, and fumarate in milk as metabolites associated with somatic cell production, wherein the concentrations of hippurate and fumarate decreased with increasing number of somatic cells [26]. In addition, choline, carnitine, citrate, and lactose in milk are known to be associated with coagulation properties [27], and citirate, $\mathrm{N}$-acetylcarbohydrates, trimethylamine, lecithin, and lactose in milk are known to be closely associated with the milk quality [28-30]. All metabolites associated with somatic cells were identified in this study, except for lactate and hippurate; further, the metabolites associated with coagulation properties were also detected. In addition, all metabolites associated with milk quality were detected except $\mathrm{N}$-acetylcarbohydrates and lecithin, and all the ketone body metabolites were observed. Therefore, milk quality testing using ${ }^{1} \mathrm{H}-\mathrm{NMR}$ spectroscopy might be a viable tool for detecting the metabolic disease of ketosis in dairy cattle.

\section{IMPLICATIONS}

Proton nuclear magnetic resonance spectroscopy and statistical analyses were employed to analyze the metabolites in dairy cattle rumen fluid and milk. The metabolites measured in the rumen fluid and milk were mostly consistent with those reported in studies conducted abroad, and may be useful for predicting metabolic diseases, and milk quality. Furthermore, this report on metabolites in ruminant biofluids, which was achieved using the proton nuclear magnetic resonance analysis, will contribute to all the future ruminal metabolism studies in Korea.

\section{CONFLICT OF INTEREST}

We certify that there is no conflict of interest with any financial organization regarding the material discussed in the manuscript.

\section{ACKNOWLEDGMENTS}

This work was carried out with the support of "Cooperative Research Program for Agriculture Science and Technology Development (Project No. PJ01503902)" Rural Development Administration, Republic of Korea.

\section{REFERENCES}

1. Xu C, Sun L, Xia C, Zhang H, Zheng J, Wang J. 1H-nuclear magnetic resonance-based plasma metabolic profiling of dairy cows with fatty liver. Asian-Australas J Anim Sci 2016; 29:219-29. https://doi.org/10.5713/ajas.15.0439

2. Nicholson JK, Lindon JC, Holmes E. 'Metabonomics': understanding the metabolic responses of living systems to pathophysiological stimuli via multivariate statistical analysis of biological NMR spectroscopic data. Xenbiotica 1999;29:11819. https://doi.org/10.1080/004982599238047

3. Tikunov AP, Johnson CB, Lee H, Stoskopf MK, Macdonald JM. Metabolomic investigations of American oysters using ${ }^{1} \mathrm{H}-\mathrm{NMR}$ spectroscopy. Mar Drugs 2010;8:2578-96. https:// doi.org/10.3390/md8102578

4. Zhu C, Li C, Wang Y, Laghi L. Characterization of yak common biofluids metabolome by means of proton nuclear magnetic resonance spectroscopy. Metabolites 2019;9:41. https://doi.org/10.3390/metabo9030041

5. Wishart DS. Metabolomics: applications to food science and nutrition research. Trends Food Sci Technol 2008;19: 482-93. https://doi.org/10.1016/j.tifs.2008.03.003

6. O'Callaghan TF, Vazquez-Fresno R, Serra-Cayuela A, et al. Pasture feeding changes the bovine rumen and milk metabolome. Metabolites 2018;8:27. https://doi.org/10.3390/metabo 8020027

7. Zhang H, Tong J, Zhang Y, Xiong B, Jiang L. Metabolomics reveals potential biomarkers in the rumen fluid of dairy cows with different levels of milk production. Asian-Australas J Anim Sci 2020;33:79-90. https://doi.org/10.5713/ajas.19.0214

8. Nyberg NT, Nielsen MO, Jaroszewski JW. Metabolic trajectories based on $1 \mathrm{H}$ NMR spectra of urines from sheep exposed to nutritional challenges during prenatal and early postnatal life. Metabolomics 2010;6:489-96. https://doi.org/10.1007/ s11306-010-0229-4

9. Klein MS, Buttchereit N, Miemczyk SP, et al. NMR metabolomic analysis of dairy cows reveals milk glycerophosphocholine to phosphocholine ratio as prognostic biomarker for risk of ketosis. J Proteome Res 2012;11:1373-81. https://doi.org/10. 1021/pr201017n

10. Yang Y, Zheng N, Zhao X, et al. Metabolomic biomarkers identify differences in milk produced by Holstein cows and other minor dairy animals. J Proteom 2016;136:174-82. https:// doi.org/10.1016/j.jprot.2015.12.031

11.Lee J, Choi M, Kang J, et al. Physicochemical, structural, pasting, and rheological properties of potato starch isolated from different cultivars. Korean J Food Sci Technol 2017;49:3608. https://doi.org/10.9721/KJFST.2017.49.4.360

12.Jung JY, Jung Y, Kim JS, Ryu DH, Hwang GS. Assessment of peeling of Astragalus roots using 1H NMR- and UPLC-MSbased metabolite profiling. J Agric Food Chem 2013;61:10398407. https://doi.org/10.1021/jf4026103 
13. Jeong JY, Hwang GS, Park JC, Kim DH, Ha M. ${ }^{1} H$ NMR-based urinary metabolic profiling of gender and diurnal variation in healthy Korean subjects. Environ Anal Health Toxicol 2010;25:295-306.

14.Jeong JY, Kim MS, Jung HJ, Kim MJ, Lee HJ, Lee SD. Screening of the liver, serum, and urine of piglets fed zearalenone using a NMR-based metabolomic approach. Korean J Agric Sci 2018;45:447-54. https://doi.org/10.7744/kjoas.20180041

15. Kim MS, Kim IY, Sung HR, et al. Metabolic dysfunction following weight regain compared to initial weight gain in a high-fat diet-induced obese mouse model. J Nutr Biochem 2019;69:44-52. https://doi.org/10.1016/j.jnutbio.2019.02.011

16. Eom JS, Lee SJ, Lee YG, Lee SS. Comparison of volatile fatty acids, monosaccharide analysis and metabolic profiling in rumen fluid according to feeding methods. J Korea Acad Ind Coop Soc 2018;19:814-24. https://doi.org/10.5762/KAIS. 2018.19.12.814

17.Eom JS, Lee SJ, Lee SK, et al. Effects of different roughage to concentrate ratios on the changes of productivity and metabolic profiles in milk of dairy cows. Korean J Org Agric 2019; 27:147-60.

18. Saleem F, Bouatra S, Guo AC, et al. The bovine ruminal fluid metabolome. Metabolomics 2013;9:360-78. https://doi.org/ 10.1007/s11306-012-0458-9

19. Eriksson L. Introduction to multi-and megavariate data analysis using projection methods (PCA \& PLS). Champaign, IL, USA: Umetrics AB; 1999.

20. Jiang G, Leem JY. Comparative analysis of cultivation region of Angelica gigas using a GC-MS-based metabolomics approach. Korean J Med Crop Sci 2016;24:93-100. https://doi.org/ 10.7783/KJMCS.2016.24.2.93

21. Nocek JE. Bovine acidosis: implications on laminitis. J Dairy Sci 1997;80:1005-28. https://doi.org/10.3168/jds.S0022-0302 (97)76026-0

22. Ametaj BN, Zebeli Q, Saleem F, et al. Metabolomics reveals unhealthy alterations in rumen metabolism with increased proportion of cereal grain in the diet of dairy cows. Metabolomics 2010;6:583-94. https://doi.org/10.1007/s11306010-0227-6

23.Wang DS, Zhang RY, Zhu WY, Mao SY. Effects of subacute ruminal acidosis challenges on fermentation and biogenic amines in the rumen of dairy cows. Livest Sci 2013;155:26272. https://doi.org/10.1016/j.livsci.2013.05.026

24. Sato H, Shiogama Y. Acetone and isopropanol in ruminal fluid and feces of lactating dairy cows. J Vet Med Sci 2010; 72:297-300. https://doi.org/10.1292/jvms.09-0227

25. Sundekilde UK, Larsen LB, Bertram HC. NMR-Based milk metabolomics. Metabolites 2013;3:204-22. https://doi.org/ 10.3390/metabo3020204

26. Sundekilde UK, Poulsen NA, Larsen LB, Bertram HC. Nuclear magnetic resonance metabonomics reveals strong association between milk metabolites and somatic cell count in bovine milk. J Dairy Sci 2013;96:290-9. https://doi.org/10.3168/jds. 2012-5819

27. Sundekilde UK, Frederiksen PD, Clausen MR, Larsen LB, Bertram HC. Relationship between the metabolite profile and technological properties of bovine milk from two dairy breeds elucidated by NMR-based metabolomics. J Agric Food Chem 2011;59:7360-7. https://doi.org/10.1021/jf202057x

28. Hu F, Furihata K, Ito-Ishida M, Kaminogawa S, Tanokura M. Nondestructive observation of bovine milk by NMR spectroscopy: analysis of existing states of compounds and detection of new compounds. J Agric Food Chem 2004;52:4969-74. https://doi.org/10.1021/jf049616o

29. Hu F, Furihata K, Kato Y, Tanokura M. Nondestructive quantification of organic compounds in whole milk without pretreatment by two-dimensional NMR spectroscopy. J Agric Food Chem 2007;55:4307-11. https://doi.org/10.1021/jf062 $803 \mathrm{x}$

30.Lamanna R, Braca A, Di Paolo E, Imparato G. Identification of milk mixtures by ${ }^{1} \mathrm{H}$ NMR profiling. Magn Reson Chem 2011;49(Suppl 1):S22-6. https://doi.org/10.1002/mrc.2807 